\title{
Acid-catalyzed Epimerization of Kobophenol A to Carasinol B
}

\section{Kejun Cheng, Gaolin Liang and Changqi Hu *}

Department of Chemistry of Natural Drugs, School of Pharmacy, Fudan University, Shanghai 200032, P. R. China; E-mails: chengkejun@gmail.com (for K. C.), stanfordliang@hotmail.com (for G. L.)

* Author to whom correspondence should be addressed; Tel: (+86)-21-54237564; Fax: (+86)-2164183411; E-mail: cqhu@shmu.edu.cn

Received: 13 March 2008; in revised form: 4 April 2008 / Accepted: 4 April 2008 / Published: 19 April 2008

\begin{abstract}
The conversion from kobophenol A into carasinol B, two main chemical constituents of Caragana sinica, was confirmed by in vitro acid-catalyzed epimerization. The result provides important information about the biotransformation of kobophenol A in plants and its metabolism in rats.
\end{abstract}

Keywords: Kobophenol A; carasinol B; Caragana sinica.

\section{Introduction}

Caragana sinica (Buc'hoz) Rehd. (Fabaceae) is widely distributed in China. Its dried roots (Chinese name: Jin-que-gen) have been used in China as a herbal medicine for the treatment of asthenia syndrome, vascular hypertension, leukorrhagia, bruises and contused wounds. In a previous study we found that the EtOAc extracts of the roots contained many oligostilbenes that had multifaceted bioactivities [1]. Kobophenol A (1), a stilbene tetramer, accounts for nearly $20 \%$ of the EtOAc extracts. Using an improved E-screen assay, we found that $\mathbf{1}$ was a phytoestrogen with estrogenic activity: it could compete with $17 \beta$-estradiol for binding to estrogen receptors, promote estrogen receptor-mediated transcription and stimulate the growth of MCF-7 and osteoblasts [2]. During the study on $C$. sinica, we found that the content of $\mathbf{1}$ decreased and that of other components increased when the extracts were stored as an alcohol syrup. This instability caused great difficulties for the quality control of this traditional Chinese herb medicine. The major component of the compounds found in the increased quantities was a new stilbene tetramer, carasinol B (2), which was first isolated, 
purified, and identified by us. As the isomer of 1, compound 2 also has stimulating activity on the proliferation of osteoblasts [3]. Since the plant extracts are weakly acidic, we reckoned that an acidic environment might be responsible for the observed transformation.

Further studies on the pharmacokinetics of $\mathbf{1}$ orally administered in rats showed that the absolute oral bioavailability of $\mathbf{1}$ was $2.0 \%$, and that 1 was mainly concentrated in the liver. These results indicated that 1 should be metabolized either by liver microsomes or by gut microflora. Based on this, we isolated new metabolites of $\mathbf{1}$ : koboquinone $\mathrm{A}$ and koboquinone $\mathrm{B}$, together with metabolite $\mathbf{2}$, from rats' feces [4]. In vitro incubation of 1 with intestinal bacteria indicated that the oxidative metabolite koboquinone A was metabolized by the intestinal bacterium Klebsiella pneumoniae [5]. Probably some other intestinal bacterium produced the other oxidative metabolite koboquinone B. However, we did not observe 2, the epimeric metabolite of $\mathbf{1}$, from intestinal bacteria. Results of these experiments hint us that the epimeric metabolite $\mathbf{2}$ in rats might be the product of $\mathbf{1}$ by the action of acidic gastric juice.

\section{Results and Discussion}

In a continuation of our efforts to study the chemical reactivity of $\mathbf{1}$ under acidic conditions, different acids including inorganic acid, organic acid and Lewis acid were examined. The reactions were monitored by HPLC analysis of total crude product mixtures obtained during each reaction. Compound 2 was obtained when 1 was treated with three different acids. Among them, $0.01 \mathrm{M} \mathrm{HCl}$ showed the best catalytic activity, while acetic acid with $\mathrm{pH} 5.0$ and the Lewis acid $\mathrm{BF}_{3}$ displayed weaker catalytic activities. The conversion rates of these reactions were $80 \%, 48 \%$ and $33 \%$, respectively. Benzylic carbons are reactive under acidic conditions. Recently Hatano et al. also reported the acid catalyzed formation of an isomeric product of (-)-epigallocatechin gallate (EGCG) [6]. In our study, ring C3 of $\mathbf{1}$ is a tetraphenol-substituted tetrahydrofuran moiety. The carbon atoms at the $7 \mathrm{c}$ and $7 \mathrm{~d}$ positions are the mostly probable to form tertiary carbocation transition states since they are benzylic carbons and connected to an oxygen atom. Therefore, under acid catalysis, the bond between atom $\mathrm{C} 7 \mathrm{c}$ and atom $\mathrm{O}$ can break, and subsequently 2 forms (Figure 1).

Figure 1. Plausible mechanisms for the conversion of 1 to 2.
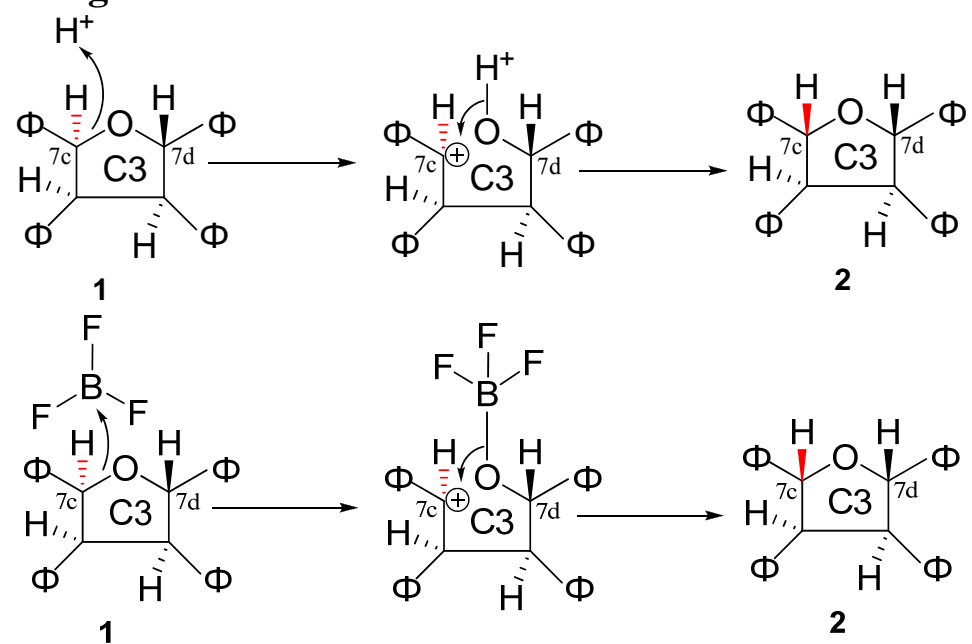
Ring A3 and ring B3 of 1 are also furan moieties, but they are benzofuran moieties. During the experiments conducted, we did not obtain any products resulting from the epimerization of these two rings. Based on the validation of the transformation above-mentioned, we suggest that 2 might be partially biosynthesized from 1 in Caragana sinica and the transformation occurs when the extracts of C. sinica are obtained as a weak acidic residue. Moreover, $\mathbf{1}$, ingested in animals, may be transformed to 2 by the action of acidic gastric juice (Figure 2).

Figure 2. Conversion from 1 to 2.

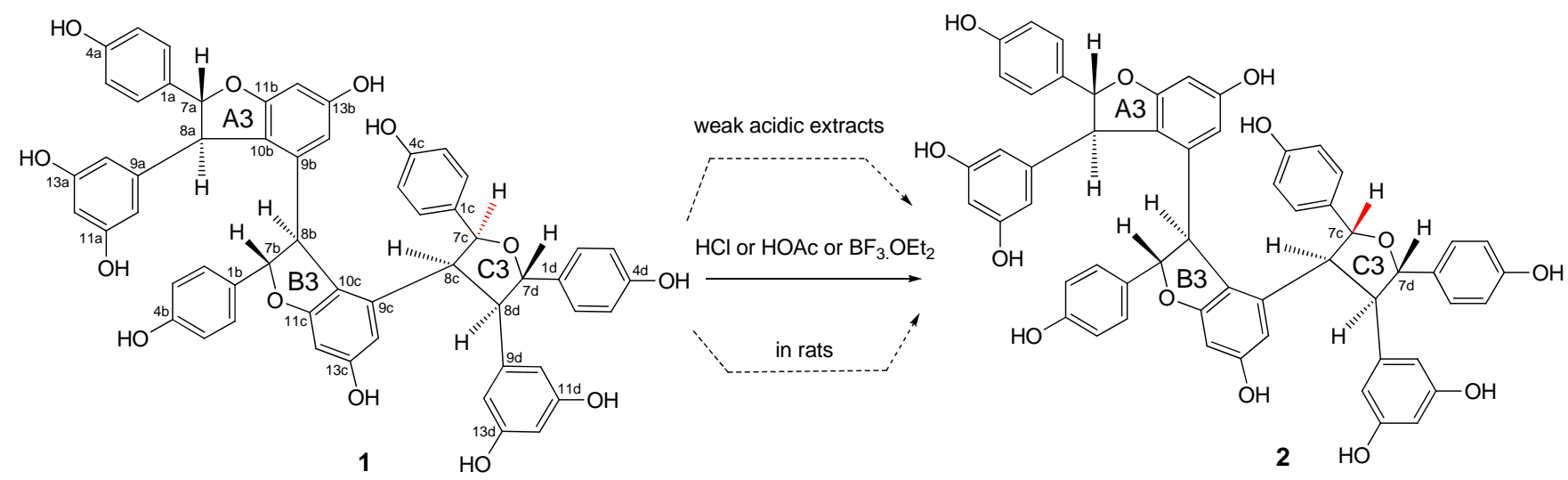

\section{Conclusions}

A tetrastilbene carasinol B (2) has been obtained from its epimer kobophenol A (1). The epimerization was catalyzed by different acids under mild conditions. The epimerization of $\mathbf{1}$ in vitro supported the hypothesis that corresponding conversion would happen in the weak acidic extracts of Caragana sinica and the acidic gastric juice when $\mathbf{1}$ were ingested by rats.

\section{Experimental}

\section{General}

UV spectra were obtained in absolute $\mathrm{MeOH}$ on a Shimadzu UV-260 spectrophotometer, IR spectra on an Avatar 360 E.S.P Fourier Transform Infrared Spectrometer, and the optical rotation on a JASCO P-1020 polarimeter in MeOH. ${ }^{1} \mathrm{H}-\mathrm{NMR},{ }^{13} \mathrm{C}-\mathrm{NMR}$ and $2 \mathrm{D}$ spectra were taken on a Mercury Plus 400 NMR spectrometer in $\mathrm{CD}_{3} \mathrm{OD}$. HRESIMS was recorded on a Q-Tof micro spectrometer. Lobar RP-18 (40-63 $\mu \mathrm{m}$, Merck) was used for column chromatography. An Agilent 1100 series chromatography system with a photodiode array detector (DAD) and an ODS-2 Hypersil C18 reversed-phase column $(5 \mu \mathrm{m}, 15 \times 0.46 \mathrm{~cm})$ were used for HPLC analysis. Kobophenol A (1) was isolated from the roots of Caragana sinica according to the literature [1]. 
General procedure for the formation of carasinol B (2) catalyzed by $\mathrm{HCl}$ or acetic acid

To a stirred solution of 1 (92.4 mg, $0.1 \mathrm{mmol})$ in methanol (3.0 mL) 0.01 M HCl (3.0 mL) (or 3.0 $\mathrm{mL}$ acetic acid with $\mathrm{pH}$ 5.0) was added at room temperature and the reaction mixture was refluxed for 18 hours. The solvent was removed under reduced pressure. The residue was then dissolved in methanol/water $(1 / 2,1 \mathrm{~mL})$ and applied to an ODS-2 RP-18 column to perform the column chromatography. This afforded $73.9 \mathrm{mg}$ of $2(80 \%)$ as pale yellow amorphous powder $(44.3 \mathrm{mg}, 48 \%$, when acetic acid with pH 5.0 was used). All the product's data including $[\alpha]$, UV, IR, ${ }^{1} \mathrm{H}-\mathrm{NMR},{ }^{13} \mathrm{C}-$ NMR and HRESIMS agreed with the previous report [3].

General procedure for the formation of carasinol $B(2)$ catalyzed by the Lewis acid $B F_{3}$

To a stirred solution of 1 (92.4 mg, $0.1 \mathrm{mmol})$ in dried acetone $(1.2 \mathrm{~mL}) 4 \AA$ molecular sieves (37.6 mg) was added at room temperature. After 10 minutes, boron trifluoride etherate $(70 \mu \mathrm{L}, 0.6$ mmol) was added in dropwise fashion, and the reaction mixture was stirred at room temperature for 18 hours. The reaction mixture was filtered and the filtration was dried under reduced pressure. The residue was then dissolved in methanol/water $(1 / 2,3 \mathrm{~mL})$ under ultrasonication and then centrifuged. The supernatant was applied to an ODS-2 RP-18 column to perform the column chromatography. This afforded $30.5 \mathrm{mg}$ of 2 (33\%).

Figure 3. HPLC Chromatograms of $\mathbf{1}$ and 2.

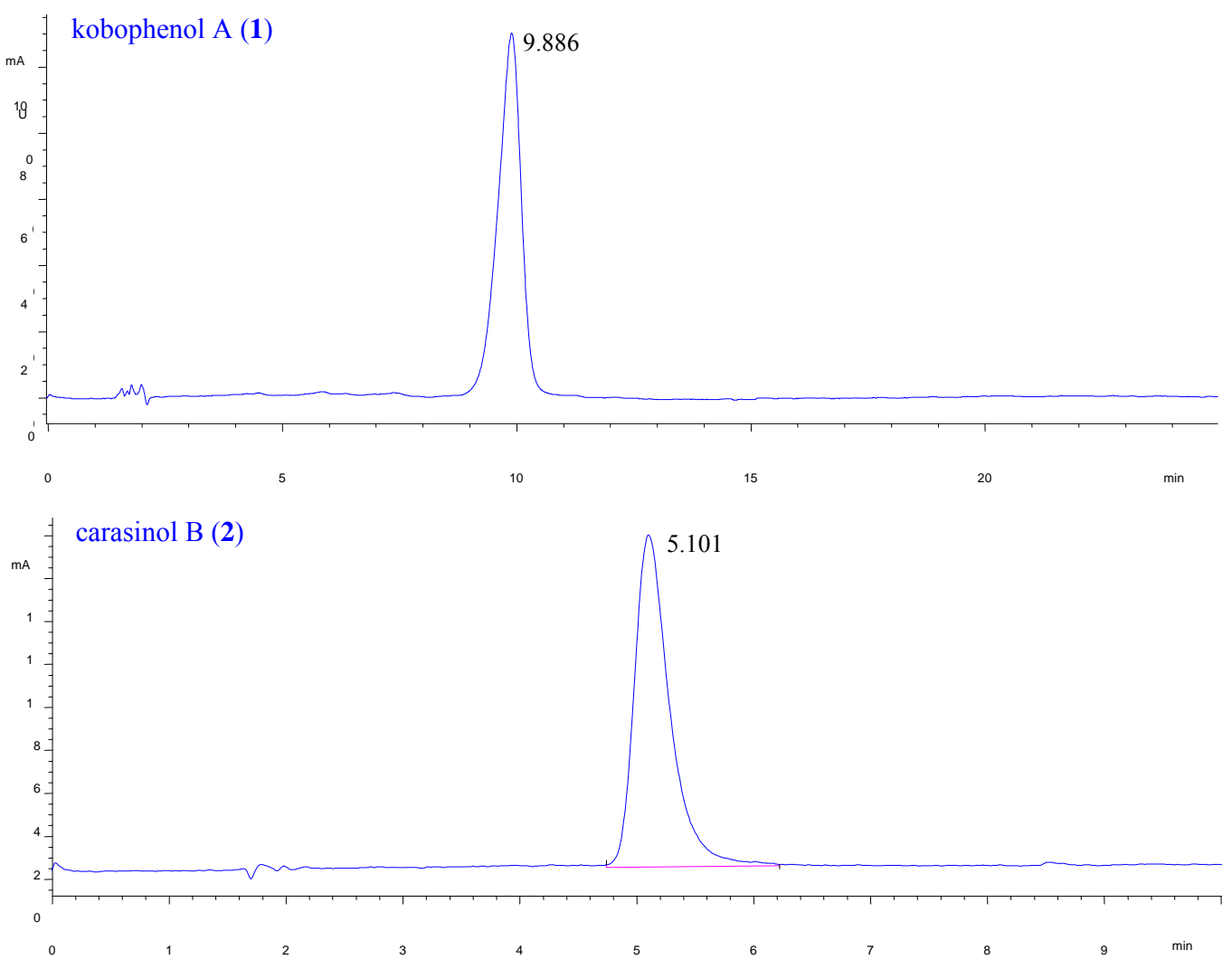


HPLC analysis of kobophenol A (1) and carasinol B (2)

The column temperature set at $30{ }^{\circ} \mathrm{C}$. The mobile phase was a mixture of $\mathrm{CH}_{3} \mathrm{CN} / \mathrm{CH}_{3} \mathrm{OH} / \mathrm{NaOAc}$ HOAc buffer ( $\mathrm{pH} 4.5,0.02 \mathrm{M}, 16: 19: 65, \mathrm{v} / \mathrm{v} / \mathrm{v})$ at a flow rate of $1.0 \mathrm{~mL} / \mathrm{min}$. The detection wavelength was set at $284 \mathrm{~nm}$. The injection volume was $20 \mu \mathrm{L}$. In the chromatograms of kobophenol A (1) and carasinol B (2, Figure 3), the two compounds show obvious retention time differences and can be easily distinguished.

\section{Acknowledgements}

This project is supported by the National Natural Science Foundation of China (No. 39270800) and the Major Subject of the Science and Technology Commission of Shanghai Municipality (No. 06DZ19709). We are grateful to Professor Peng Xia of our University for his instruction in synthetic experiments. Thanks also to Professor Caiyun Liu for her contributions to the NMR measurements.

\section{References}

1. Luo, H.F.; Zhang, L.P.; Hu, C.Q. Five novel oligostilbenes from the roots of Caragana sinica. Tetrahedron 2001, 57, 4849-4854.

2. Tian, C.Y.; Hu, C.Q.; Xu, G.; Song, H.Y. Assessment of estrogenic activity of natural compounds using improved E-screen assay. Acta. Pharmacol. Sin. 2002, 23, 572-576.

3. Ma, D.Y.; Luo, H.F.; Hu, C.Q. Three stilbene tetramers from the roots of Caragana sinica. Chin. J. Chem. 2004, 22, 207-211.

4. Liang, G.L.; Cheng, K.J.; Zhou, H.; Hu, C.Q. Koboquinone A and B, new metabolites of kobophenol A in rat. Chem. Pharm. Bull. 2004, 52, 1489-1491.

5. Liang, G.L.; Qian, L..S.; Cui, Y.L.; Hu, C.Q. Formation of oxidative metabolite from kobophenol A by human intestinal bacterium Klebsiella pneumonia. Chem. Biodivers. 2005, 2, 506-509.

6. Hatano, T.; Hori, M.; Kusuda, M.; Ohyabu, T.; Ito, H.; Yoshida, T. Characterization of the oxidation products of (-)-epigallocatechin gallate, a bioactive tea polyphenol, on incubation in neutral solution. Heterocycles 2004, 63, 1547-1554.

Sample Availability: Samples of compounds $\mathbf{1}$ and $\mathbf{2}$ are available from the authors.

(C) 2008 by MDPI (http://www.mdpi.org). Reproduction is permitted for noncommercial purposes. 